\title{
A Model-Based Decoupling Method for Surge Speed and Heading Control in Vessel Path Following
}

\author{
Xudong Wang, ${ }^{1}$ Jin Zhao ${ }^{1},{ }^{1}$ and Tao Geng ${ }^{2}$ \\ ${ }^{1}$ Guangdong HUST Industrial Technology Research Institute, \\ Guangdong Province Key Laboratory of Digital Manufacturing Equipment, \\ Key Laboratory of Image Processing and Intelligent Control of the Ministry of Education of China, \\ School of Artificial Intelligence and Automation, Huazhong University of Science and Technology, Wuhan 430074, China \\ ${ }^{2}$ Guangdong HUST Industrial Technology Research Institute, Dongguan 523000, Guangdong, China \\ Correspondence should be addressed to Jin Zhao; jinzhao617@163.com
}

Received 16 March 2020; Accepted 27 June 2020; Published 22 July 2020

Guest Editor: Xiao Ling Wang

Copyright (C) 2020 Xudong Wang et al. This is an open access article distributed under the Creative Commons Attribution License, which permits unrestricted use, distribution, and reproduction in any medium, provided the original work is properly cited.

In this paper, to solve the surge speed loss problem generated by sway-yaw motion in the path-following control, a model-based decoupling (MBD) method for surge speed and heading control in vessel path following is proposed. The guidance law is designed independently in the kinematic level. In the kinetic level, the surge model and sway-yaw model can be decoupled by assuming that the surge speed varies slowly, and the heading controller and surge speed controller are designed under the framework of the MBD method. Commonly, the surge speed controller is ignored in the path following or designed separately. In the MBD method, the heading controller is designed first through the MPC method, and the coupling terms between the surge model and sway-yaw model are treated as time-varying disturbances, which can be predicted through the outcomes of the heading controller. Then, the time-varying disturbances are compensating in the surge speed controller so that the surge speed can be feedforward compensated to achieve better performance. The simulation results compared the surge speed performance in path following of the MBD method and usual approaches to illustrate the effectiveness of the MBD method.

\section{Introduction}

Unmanned Aerial Vehicle (UAV), Unmanned Ground Vehicle (UGV), and Unmanned surface vessel (USV) have attracted more and more attention in recent years. The unmanned agents have great potentials in applications such as patrol, hydrologic exploration, and transportation when they are cooperative as multiagent systems. The coordinated control for multiagent systems has received increasing attention recently [1-6], also the control of single agent is essential to the whole multiagent system. Path following is a very crucial technology for Unmanned surface vessels (USVs) sailing in the ocean; many applications require that the USV s have the capability to drive to the task area along a predefined path. The purpose of the path following controller is to minimize the error between the real position of USVs and the predefined path in vessel sailing. The path guidance laws designed at the kinematic level can be used for way-point path following [7-9] and curve path following [10-14]. For example, autopilot is a kind of path-following controller at the kinematic level which has been wildly used in ocean transportation.

Many other methods which combine the kinematic model and kinetics model are also proposed to achieve the goal of path following. Some robust nonlinear methods are used to force the vessel to follow the predefined path. In [15], Lyapunov's direct approach and backstepping method are used to design the controller. In [16], several coordinate transformations and the backstepping technique are used to design a global controller for vessel path following. In [17], the backstepping nonlinear controller design is based on feedback dominance, and the experiment results demonstrate the effectiveness of the proposed method. In [18], the path following controller design is divided into two parts via 
backstepping technique. In [19], a backstepping-based pathfollowing control algorithm is presented to ensure that the tracking errors of the surface vessel remain within the required performance constraints. In [20], a neural sliding mode controller is designed for vessel path following in the external disturbance and parameter perturbation. In [21, 22], the stability of integral line-of-sight (ILOS) guidance method for a path-following system of underactuated marine vehicles is analyzed. In order to reduce the large cross-track error in turning by adjusting vessel speed, an inverse optimal control method is proposed in [23]. In [24], a reference governor is used for generating the optimal reference signals within the state and input constraints, and the optimization problem is solved by a projection neural network.

It is a challenge to address the actuator saturation and state constraints in controller design for robust nonlinear methods. In [25], the error constraint of the vessel position is handled by integrating a novel tan-type barrier Lyapunov function. However, how to handle lots of different actuators and state constraints is still difficult in the robust method. Due to the advantage of dealing with constraints, many methods under the framework of MPC are proposed to solve path-following problems. In [17], the path-following problem is solved by using a linear MPC controller with rudder and roll constraints. In [26], an LOS decision variable can be incorporated into the MPC design. The lookahead distance is optimized in solving the MPC problem so that the path following has a better performance than the constant lookahead distance LOS method. In [27], a nonlinear statespace model consists of a path following error model and a kinetic model as the tracking model is established; then, a nonlinear MPC controller is designed. In [28], a robust MPC method is proposed for vessel path following control under the constraints of the rudder angle and roll angle. In [29], a nonlinear MPC controller is designed to solve the combined path planning and tracking control problem for an AUV.

In the application of the way-point path following, surge speed control is essential since time constraint is a condition that cannot be ignored. In [7], the speed is controlled through the state feedback linearization method. In [30], different approaches such as proportional-integral and feedback linearization are tested in vessel speed control experiments. Except for designing a speed controller independently, the speed and yaw subsystem can be designed together. In [31], a dynamic window-based controller is used to control the surge speed under the actuator constraints. In [32], the speed and yaw subsystem are controlled through the backstepping and Lyapunov method.

In this paper, to improve the speed and heading controller performance in path following, a model-based decoupling (MBD) method is proposed. The heading controller is designed first under the framework of MPC; then, the coupling terms between the surge model and yaw subsystem are predicted through the output of heading controller. In the speed MPC controller, the coupling terms are compensated as a time-varying disturbance. The speed and heading control of the vessel using a kinetic model is a nonlinear control problem under the framework of MPC. By using the MBD method, the original nonlinear MPC control problem breaks down into two low-dimensional nonlinear MPC problem or two linear MPC problem.

The organization of this paper is as follows. Section 2 states the problem formulation. Section 3 presents the design of the path following guidance laws and MBD method. Section 4 illustrates and analyzes the simulation results. Section 5 summarizes conclusions.

\section{Problem Formulation}

2.1. Mathematical Model for Vessel Path Following. In this section, the kinematic and kinetic model of USVs is introduced first. The vessel model normally is a six degree-offreedom (DOF) nonlinear model, including surge, lateral, vertical velocity, and pitch, roll, and yaw angle. The states in 6-DOF model such as pitch angle and roll angle can be neglected in path-following control, so, the kinematic model in north-east coordinate such as equation (1a) and the 3DOF kinetic model proposed in [33] such as equation (1b), are used here to describe path following:

$$
\begin{gathered}
\dot{\eta}=R(\eta) \nu, \\
M \dot{v}+C(\nu) \nu+D(\nu) \nu=B \tau+\tau_{\text {wind }}+\tau_{\text {wave }}
\end{gathered}
$$

where the vector $\eta=\left[\begin{array}{lll}x & y & \psi\end{array}\right]^{\mathscr{T}}$ denotes the position and yaw angle in the north-east coordinate and $R(\eta)$ is the rotation matrix. The detail form of equation (1a) is

$$
\begin{aligned}
& \dot{x}=u \cos (\psi)-v \sin (\psi), \\
& \dot{y}=u \sin (\psi)+v \cos (\psi), \\
& \dot{\psi}=r,
\end{aligned}
$$

where $v=\left[\begin{array}{lll}u & v & r\end{array}\right]^{\mathscr{T}}$ denotes the surge velocity, lateral velocity, and yaw angle velocity in the body-fixed coordinate. Vector $\tau=\left[\begin{array}{lll}\tau x & 0 & \tau z\end{array}\right]^{\mathscr{T}}$ denotes actuator force and torques, $\tau_{\text {wind }}$ denotes force and torques caused by wind, $\tau_{\text {wave }}$ denotes force and torques caused by the wave, $M$ accounts for inertial effects, matrix $C(\nu)$ accounts for centrifugal and Coriolis effects, and matrix $D(\nu)$ accounts for viscous and dissipative effects. The exact expression of the matrix is

$$
\begin{aligned}
M & =\left[\begin{array}{ccc}
m-X \dot{u} & 0 & 0 \\
0 & m-Y_{\dot{v}} & m X_{g}-Y_{\dot{r}} \\
0 & m X_{g}-N_{\dot{v}} & I_{z}-N_{\dot{r}}
\end{array}\right], \\
C(v) & =\left[\begin{array}{ccc}
0 & 0 & c 13 \\
0 & 0 & c 23 \\
-c 13 & -c 23 & 0
\end{array}\right], \\
D(v) & =\left[\begin{array}{ccc}
d 11 & 0 & 0 \\
0 & d 22 & d 23 \\
0 & d 32 & d 33
\end{array}\right],
\end{aligned}
$$

where 


$$
\begin{aligned}
& c 13=-m\left(X_{g} r+v\right)+Y_{\dot{v}} v+\frac{1}{2}\left(Y_{\dot{r}}+N_{\dot{v}}\right) r, \\
& c 23=m u-X_{\dot{u} u}, \\
& d 11=-X_{u}-X_{|u| u}|u|-X_{u u} u^{2}, \\
& d 22=-Y_{v}-Y_{|v| v}|v|-Y_{|r| v}|r|, \\
& d 23=-Y_{r}-Y_{|v| r}|v|-Y_{|r| r}|r|, \\
& d 32=-N_{v}-N_{|v| v}|v|-N_{|r| r \mid}|r|, \\
& d 33=-N_{r}-N_{|v| r}|v|-N_{|r| r}|r| .
\end{aligned}
$$

The vessel named CyberShip II in [33] has two main propellers, two rudders aft, and one bow thruster fore. For simple but without loss of generality, the vessel model of CyberShip II used in this paper has no bow thruster, but one propeller and one rudder. The surging force generated by one propeller is equal to two propellers of CyberShip II, and so is the steering moment. The exact expression of actuator configuration matrix $B$ is

$$
B=\left[\begin{array}{cc}
2 & 0 \\
0 & 2 \\
0 & -\left|l_{x} R_{1}\right|-\left|l_{x} R_{2}\right|
\end{array}\right] .
$$

The kinetic model can be rewritten as $\dot{v}=-M^{-1} C(\nu) \nu+$ $-M^{-1} D(\nu) \nu+M^{-1} B \tau$. The detail form of the kinetic model is

$$
\begin{aligned}
& \dot{u}=a_{1} u+a_{2} u^{2}+a_{3} u^{3}+a_{4} v r+a_{5} r^{2}+a_{6} u^{2} \delta^{2}+a_{7} \tau_{x}, \\
& \dot{v}=b_{1} u v+b_{2} u r+b_{3} v|v|+b_{4} v|r|+b_{5} r|v|+b_{6} r|r|+b_{7} \tau_{z}, \\
& \dot{r}=c_{1} u v+c_{2} u r+c_{3} v|v|+c_{4} v|r|+c_{5} r|v|+c_{6} r|r|+c_{7} \tau_{z} .
\end{aligned}
$$

2.2. Path-following Formulation. The guidance law used in this paper is designed at the kinematic level. Usually, the $C^{1}$ parametrized path $\left(x_{p}(\theta), y_{p}(\theta)\right)$ with $\theta \geq 0$ are used to denote path including curve path or way-point path. In this paper, the cross-error of the path following is our concern, and the expected surge speed is given by manual. Since many path-following guidance laws are valid in both curve path following and way-point path following, the situation of the way-point path following is only considered in this paper for simplicity. For a vessel located at $(x, y)$, the orthogonal distance $y_{e}$ to the path can be calculated by equation (7) proposed in [10]:

$$
y_{e}=-\left(x-x_{p}\right) \sin (\theta)+\left(y-y_{p}\right) \cos (\theta),
$$

where $\theta$ is the slop of straight line between two way-point $\left(x_{i}, y_{i}\right)$ and $\left(x_{i+1}, y_{i+1}\right)$, for $i=1,2, \ldots, N$, and $\theta$ can be calculated by $\theta=\tan ^{-1}\left(y_{i+1}-y_{i} / x_{i+1}-x_{i}\right)$. The cross point $\left(x_{p}, y_{p}\right)$ can be calculated by the following equation.

$$
\left\{\begin{array}{l}
y_{i+1}-y_{p}=\tan (\theta)\left(x_{i+1}-x_{p}\right), \\
y-y_{p}=-\frac{1}{\tan (\theta)}\left(x-x_{p}\right), \quad \theta \neq 0 \text { and } \theta \neq \frac{\pi}{2},
\end{array}\right.
$$

when $\theta=0$, cross point $\left(x_{p}, y_{p}\right)=\left(x, y_{i+1}\right)$, and when $\theta=\pi / 2$, cross point $\left(x_{p}, y_{p}\right)=\left(x_{i+1}, y\right)$.

Equation (7) is differentiable, and the differential form is

$$
\begin{aligned}
\frac{\partial y_{e}}{\partial \psi}= & -(u \cos (\psi)-v \sin (\psi)) \sin (\theta)+(u \sin (\psi) \\
& +v \cos (\psi)) \cos (\theta)
\end{aligned}
$$

Equation (9) can be written in a more compact form as

$$
\dot{y}_{e}=U \sin (\psi-\theta+\beta) \text {, }
$$

where $U=\sqrt{u^{2}+v^{2}}$ and $\beta=\tan ^{-1}(v / u)$ is the speed sideslip angle. In this paper, all the states including sideslip assumption can be measured directly. Assuming that sideslip angle $\beta$ is small and constant implies $\cos (\beta) \approx 1$ and $\sin (\beta) \approx \beta$. Equation (10) can be simplified as

$$
\dot{y}_{e}=U \sin (\psi-\theta)+U \cos (\psi-\theta) \beta \text {. }
$$

\section{MBD Method for Surge Speed and Heading Control}

In this section, the path-following guidance law is introduced first, since the focus of this paper is the MBD method, and the path-following guidance law equation (12) proposed in [10] is used in this paper to generated reference heading $\psi_{d}$; the stability of the guidance laws can be referred to [10]

$$
\psi_{d}=y_{p}+\tan ^{-1}\left(\frac{1}{\Delta} y_{e}+\beta\right)
$$

Robust nonlinear methods such as the backstepping method and direct Lyapunov method can be used to design surge speed and heading controller based on the nonlinear 3-DOF maneuvering model. However, these methods cannot handle the constraints of state and actuator. Also, it is a challenge to design such a controller when the maneuvering model is complex.

The nonlinear MPC method based on the 3-DOF maneuvering model can be used to control heading and surge speed together under the constraints [34]; the solution of the nonlinear MPC problem can only be solved through numerical iteration methods such as Gauss-Newton method. All solutions based on gradient iteration method can only guarantee the local minimum, also it is a huge computation burden for on-board computer in real-time control.

To reduce the complexity of designing controller, usually, the surge speed controller and heading controller are designed independently due to which the 3-DOF maneuvering model can be decoupled in a forward speed (surge) model and a sway-yaw subsystem for maneuvering [35]. The 
independent surge speed and heading controller both have good performance when the reference heading and reference speed are steady. However, there is an inevitable speed loss when there is sway-yaw motion.

The MBD method for surge speed and heading control are designed under the framework of MPC. Figure 1 shows the guidance and control architecture for vessel path following. The MPC heading controller is designed independently based on the sway-yaw model linearized at a given work point. The MPC surge speed controller is also based on the linearized surge model. The time-varying added resistance generated by sway-yaw motion is only considered in the process of solving the optimal sequence, which does not add much computation burden. The nonlinear MPC problem breaks down into two low-dimensional linear MPC problem through the MBD method. Since the heading controller and the surge speed controller are designed independently, it is much more convenient for parameters turning in the controller design process.

During every MBD method control interval, the MPC heading controller is solved fist. Then, the coupling terms of the surge model are calculated through the outcome sequence of the MPC heading controller, and these coupling terms are treated as time-varying added resistance. Next, the MPC surge model is solved considering the time-varying added resistance. Finally, the first element of the heading controller outcome sequence and the speed controller outcome sequence are used as controller output.

The rest of this section now focuses on the derivation of the MBD method.

3.1. Heading Controller. In this section, the MPC heading controller with actuator constrains is first designed. The purpose of the heading controller is to steer the vessel heading to the excepted heading $\psi_{d}$.

During the path-following task, the surge speed is controlled by a speed controller, so we assume that the surge speed is constant during every control interval, and the sway-yaw subsystem equations (6b) and (6c) can be decoupled from an original nonlinear system. The linear state-space model is derived by linearizing the sway-yaw subsystem at a given work point to reduce the complexity of designing the controller. Assuming surge speed $u=u_{o}$, lateral velocity $v \approx 0$, yaw angular velocity $r \approx 0$, and the right hand of equations (6b) and (6c) can express in a Taylor series by taking the partial derivative. By retaining the firstorder terms of Taylor series and incorporating equation with $\dot{\psi}=r$, the linearized sway-yaw subsystem can be written as

$$
\dot{x}=A_{c} x+B_{c} \tau z,
$$

where $\dot{x}=\left[\begin{array}{c}\dot{v} \\ \dot{r} \\ \dot{\psi}\end{array}\right], A_{c}=\left[\begin{array}{ccc}b_{1} u & b_{2} u & 0 \\ c_{1} u & c_{2} u & 0 \\ 0 & 1 & 0\end{array}\right]$, and $B_{c}=\left[\begin{array}{c}b_{7} \\ c_{7} \\ 0\end{array}\right]$.

The controllers are designed in a discrete form; the heading model can be simplified to a first-order transfer

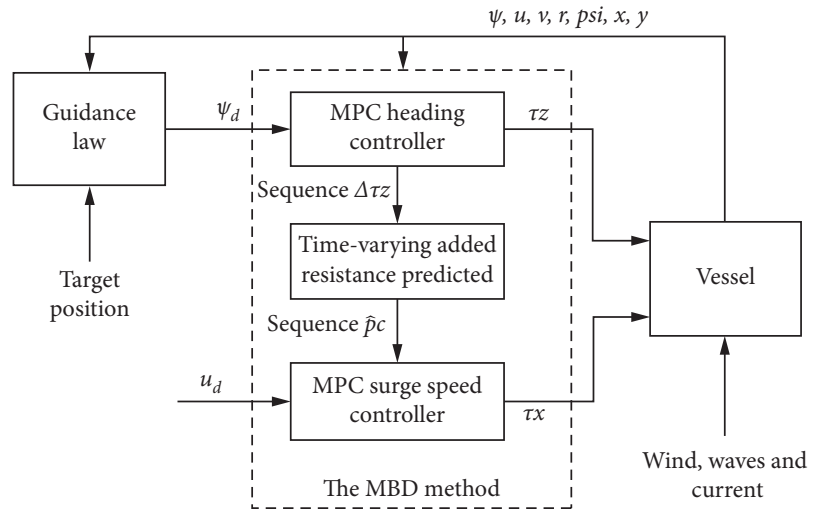

FIgURE 1: Architecture of the MBD method.

function model. Normally, the sampling interval $T_{s}$ is chosen as one-tenth of the time constant in the simplified first-order function model. When the sampling interval $T_{s}$ is given, the discreet form of equation (13) can be easily calculated by the following transform:

$$
x_{k+1}=A_{d} x_{k}+B_{d} \tau z_{k},
$$

where $x_{k+1}=\left[\begin{array}{lll}v_{k+1} & r_{k+1} & \psi_{k+1}\end{array}\right]^{\mathscr{T}}, A_{d}=e^{A_{c} T_{s}}$, and $B_{d}=$ $\int_{0}^{T_{s}} e^{A_{c} T_{s}} \mathrm{~d} t B_{c}$. Due to the effect of wind, current, wave, and unknown disturbance, the predicted progress incorporating the external disturbance $d$ is used to correct the prediction results at every control interval. For the simplicity of solving online optimization, equation (14) is written in the augmented form as

$$
\begin{gathered}
z_{k+1}=A z_{k}+B \Delta \tau z_{k}+d_{k}, \\
\psi_{k+1}=C z_{k+1},
\end{gathered}
$$

where $\quad z_{k+1}=\left[\begin{array}{c}v_{k+1} \\ r_{k+1} \\ \psi_{k+1} \\ \tau z_{k+1}\end{array}\right], \quad A=\left[\begin{array}{cc}A_{d} & B_{d} \\ 0 & I\end{array}\right], \quad B=\left[\begin{array}{c}B_{d} \\ I\end{array}\right]$, $C=\left[\begin{array}{llll}0 & 0 & 1 & 0\end{array}\right]$, and $I$ is the identity matrix.

As we all know, the energy output by the physical system is not infinite, and the torque force generated by vessel actuators such as a rudder or water-jet system has the torque rate constraints and bounded value constraints. So, the constraints of the actuator are considered in the heading control online optimal progress, $\Delta \underline{\tau z}$ and $\Delta \overline{\tau z}$ are the lower and upper bounds of the $\tau z$ varying rate, respectively, and $\underline{\tau z}$ and $\overline{\tau z}$ are the lower and upper bounds of the $\tau z$ value, respectively.

The cost function consists of two quadratic terms, the heading error quadratic term grantee that the real heading convergence to the reference heading and the rudder varying rate quadratic term can reduce the rudder movement. Then, the heading control problem is transformed into a constrained online optimization problem as equation (16), $N_{p}$ is the prediction horizon, and $N_{u}$ is the control horizon: 


$$
\begin{gathered}
\min \quad J\left(z_{k}, \Delta \tau z\right)=\sum_{j=1}^{N_{p}}\left(C z_{k+j}-\psi_{d}\right)^{T} \mathrm{Q}\left(C z_{k+j}-\psi_{d}\right) \\
+\sum_{j=1}^{N_{u}} \Delta \tau z_{k+j}^{T} R \Delta \tau z_{k+j}^{T},
\end{gathered}
$$

which subjects to

$$
\begin{gathered}
\Delta \underline{\tau z} \leq \Delta \tau z_{k+j-1} \leq \Delta \overline{\tau z}, \quad j=1,2, \ldots, N_{u}, \\
\underline{\tau z} \leq \tau z_{k+j} \leq \overline{\tau z}, \quad j=1,2, \ldots, N_{u} .
\end{gathered}
$$

The online optimization problem equation (16) can be transformed into a quadratic problem to solve the optimal solution sequence $\Delta \tau \mathbf{Z}^{*}=\left\{\Delta \tau z_{k}^{*}, \Delta \tau z_{k+2}^{*}, \ldots, \Delta \tau z_{k+N_{u}-1}^{*},\right\}$ at each time $k$. According to equations (15a) and (15b), the state prediction with prediction horizon $N_{p}$ and control horizon $N_{u}$ can be written as

$$
\psi_{\text {pre }}=P z_{k}+H \Delta \tau \mathbf{z}+E d_{k},
$$

where $\quad \psi_{\text {pre }}=\left[\begin{array}{c}\psi_{k+1} \\ \psi_{k+2} \\ \vdots \\ \psi_{k+N_{p}}\end{array}\right], \quad P=\left[\begin{array}{c}C A \\ C A^{2} \\ \vdots \\ C A^{N_{p}}\end{array}\right]$,

$H=$

$\left[\begin{array}{cccc}C B & 0 & \cdots & 0 \\ \vdots & \vdots & & \vdots \\ C A^{N_{u}-1} B & C A^{N_{u}-2} B & \cdots & B \\ \vdots & \vdots & & \vdots \\ C A^{N_{u}-1} B & C A^{N_{u}-2} B & \cdots & B\end{array}\right]$, and $E=\left[\begin{array}{c}C \\ C A+I \\ \vdots \\ \sum_{j=0}^{N_{p}-1} C A^{j}\end{array}\right]$. The

disturbance at time $k$ is updated by the error of real state and predicted state as

$$
d_{k}=z_{k}-\left(A z_{k-1}+B \Delta \tau z_{k-1}\right) .
$$

Substituting equation (18) into equation (16) and ignoring the constant value terms which do not affect the solution of solving the optimization problem, the cost function can be written as a quadratic form:

$$
\min \quad J\left(z_{k}, \Delta \tau \mathbf{z}\right)=\frac{1}{2} \Delta \tau \mathbf{z}^{T} S \boldsymbol{\Delta} \tau \mathbf{z}+f^{T} \boldsymbol{\Delta} \tau \mathbf{z} .
$$

Matrix $S=H^{T} Q H+R$ is the quadratic objective term, and matrix $f=H^{T} Q\left(P z_{k}+E d_{k}-\psi_{\mathbf{d}}\right)$ is the linear term. The constrained quadratic programming can be solved by the numerical method. The controller output $\tau z_{k}=\tau z_{k-1}+\Delta \tau z_{k}$, and $\Delta \tau z_{k}$ is the first element of $\Delta \tau \mathbf{z}^{*}$. The stability of the MPC controller without terminal constraints can be guaranteed by choosing a large prediction horizon [36].

3.2. Surge Speed Controller. In this section, the MPC surge speed controller with surge force constraints is designed. Under the framework of MPC, the constraints of the state and actuator can be handled easily. The standard MPC (SMPC) method is first introduced to the design surge speed controller; then, the surge speed controller based on the MBD method is introduced. The discredited linear surge model equation (21) can be derived by linearizing the nonlinear surge model equation (6a) at a given work point:

$$
u_{k+1}=A_{1} u_{k}+B_{1} \tau x,
$$

where $A_{1}=e^{a_{1} T_{s}}$ and $B_{1}=\int_{0}^{T_{s}} e^{a_{1} T_{s}} \mathrm{~d} t a_{7}$.

When a vessel is in the surge motion, yawing motion due to steering can result in added resistance in calm water as well as yawing due to wave motion [37], and the added resistance can significantly reduce surge speed. Usually, the surge speed controller is designed independently based on the linear surge model equation (21) through the standard MPC method and all the disturbance, including the added resistance and the effect of the wave, wind, and current are treated as an unknown disturbance.

The derivation of the SMPC method is introduced as follows. Considering the unknown disturbance $\omega$ and rewriting the sure model equation (21) as augmented for simplicity, the modified surge model is

$$
\begin{gathered}
y_{k+1}=A_{u} y_{k}+B_{u} \tau x_{k}+d u_{k}, \\
u_{k+1}=C_{u} y_{k+1},
\end{gathered}
$$

where $\quad y_{k+1}=\left[\begin{array}{c}u_{k+1} \\ \tau x_{k+1}\end{array}\right], \quad A_{u}=\left[\begin{array}{cc}A_{1} & B_{1} \\ 0 & 1\end{array}\right], \quad B_{u}=\left[\begin{array}{c}B_{1} \\ 1\end{array}\right]$, $d u_{k}=\left[\begin{array}{c}\omega_{k} \\ 0\end{array}\right]$, and $C_{u}=\left[\begin{array}{ll}1 & 0\end{array}\right]$. The disturbance $\omega_{k}$ at time $k$ is updated by the error of real surge speed and predicted surge speed as

$$
\omega_{k}=u_{k}-\left(A_{1} u_{k-1}+B_{1} \Delta \tau x_{k-1}\right) .
$$

Then, the augmented surge model is used to construct a MPC controller.

Based on the modified surge model, the surge speed control problem with excepted surge speed $\mathbf{u}_{\mathbf{d}}$ now is an online optimization problem as cost function equation (24). The speed error quadratic term in equation (24) grantee that the real speed convergence to the reference speed and the force varying rate quadratic term in equation (24) can reduce the force varying. The first element of optimal solution sequence $\Delta \tau \mathbf{x}^{*}=\left\{\Delta \tau x_{k}^{*}, \Delta \tau x_{k+2}^{*}, \ldots, \Delta \tau x_{k+N_{u}-1}^{*},\right\}$ is the surge force increment at each time $k$. The prediction horizon is $N_{p u}$, and control horizon is $N_{u u}$ :

$$
\begin{gathered}
\min J\left(y_{k}, \Delta \tau x\right)=\sum_{j=1}^{N_{p u}}\left(C_{u} y_{k+j}-u_{d}\right)^{T} Q\left(C_{u} y_{k+j}-u_{d}\right) \\
+\sum_{j=1}^{N_{u}} \Delta \tau x_{k+j}^{T} R \Delta \tau x_{k+j}^{T},
\end{gathered}
$$

which subjects to

$$
\begin{gathered}
\Delta \underline{\tau x} \leq \Delta \tau x_{k+j-1} \leq \Delta \overline{\tau x}, \quad j=1,2, \ldots, N_{u u}, \\
\underline{\tau x} \leq \tau x_{k+j} \leq \overline{\tau x}, \quad j=1,2, \ldots, N_{u u} .
\end{gathered}
$$

The drawback of the SMPC is that there will be no compensating force of disturbance until there is an offset in 
surge speed since the process of updating disturbance $\omega$ at time $k$ always occurs at the next control interval. The MBD method is proposed in this paper to overcome the delay in updating disturbance. In the MBD method, the sway-yaw motion due to the steering is predicted by the actuator sequence $\Delta \tau \mathbf{z}^{*}$ and compensated as a time-varying disturbance $\widehat{\mathbf{p}} \mathbf{c}=\left\{\widehat{p} c_{k}, \widehat{p} c_{k+1}, \ldots, \widehat{p} c_{k+N_{p d}-1}\right\}$ in forward speed control. The calculation of $\widehat{\mathbf{p c}}$ involves several steps, as described in the sequel:

(1) At time $k$, calculate the predicted sequence of $\widehat{\mathbf{v}}, \widehat{\mathbf{r}}$ through equations (15a) and (15b) by using $\Delta \tau \mathbf{z}^{*}$.

(2) Calculate the time-varying disturbance $\widehat{p} c_{k}$ by using part terms of equation (6a), $\widehat{p}_{k}=a_{4} v_{k} r_{k}+a_{5} r_{k}^{2}$. The states $v_{k}$ and $r_{k}$ are measured directly at present time $k$.

(3) Calculate the rest of time-varying disturbance sequence by using part terms of equation (6a), $\widehat{p} c_{k+1}=a_{4} \widehat{v}_{k+1} \widehat{r}_{k+1}+a_{5} \widehat{r}_{k+1}^{2}$. The predicted state $\widehat{v}_{k+j}$ and $\widehat{r}_{k+j}, j=1, \ldots, N_{p d}-1$, are calculated at step 1 .

Substituting $\widehat{\mathbf{p}} \mathbf{c}$ into equations (22a) and (22b), the surge model is modified as follows:

$$
\begin{aligned}
& y_{k+1}=A_{u} y_{k}+B_{u} \tau x_{k}+d u_{k}, \\
& u_{k+1}=C_{u} y_{k+1}+\widehat{p} c_{k},
\end{aligned}
$$

and the equation of updating $\omega_{k}$ is modified as

$$
\omega_{k}=u_{k}-\left(A_{1} u_{k-1}+B_{1} \Delta \tau x_{k-1}+\widehat{p} c_{k-1}\right) .
$$

The cost function based on equations (26a) and (26b) and equation (27) can be written as

$$
\begin{gathered}
\min \quad J\left(y_{k}, \Delta \tau x\right)=\sum_{j=1}^{N_{p u}}\left(C_{u} y_{k+j}+\widehat{p} c_{k+j-1}-u_{d}\right)^{T}, \\
Q\left(C_{u} y_{k+j}+\widehat{p} c_{k+j-1}-u_{d}\right)+\sum_{j=1}^{N_{u}} \Delta \tau x_{k+j}^{T} R \Delta \tau x_{k+j}^{T},
\end{gathered}
$$

which subjects to

$$
\begin{gathered}
\Delta \underline{\tau x} \leq \Delta \tau x_{k+j-1} \leq \Delta \overline{\tau x}, \quad j=1,2, \ldots, N_{u u}, \\
\underline{\tau x} \leq \tau x_{k+j} \leq \overline{\tau x}, \quad j=1,2, \ldots, N_{u u} .
\end{gathered}
$$

Also, the online optimization problem equations (25a), (25b), and (28) can be transformed into constrained quadratic programming, the controller output $\tau x_{k}=\tau x_{k-1}+$ $\Delta \tau x_{k}$, and $\Delta \tau x_{k}$ is the first element of $\Delta \tau \mathbf{x}^{*}$. The stability of the MPC controller can be guaranteed by choosing a large prediction horizon $N_{p u}$. Since the focus of this paper is the performance of the MBD method, the detailed proof of MPC stability can be referred to [36].

\section{Simulation Results}

In this section, the results of the way-point path following are presented. The simulation results of the MBD method and the standard MPC (SMPC) method are compared to illustrate the
TABLE 1: The parameters of the vessel.

\begin{tabular}{lc}
\hline$m$ & 23.8 \\
Iz & 17.6 \\
xg & 0.046 \\
Xudot & -2.0 \\
Xu & -0.72253 \\
Xabsuu & -1.32742 \\
Xuuu & -5.86643 \\
Yvdot & -10.0 \\
Yrdot & -0.0 \\
Yv & -0.88965 \\
Yabsvv & -36.47287 \\
Yabsrv & -0.805 \\
Yr & -7.250 \\
Yabsvr & -0.845 \\
Yabsrr & -3.450 \\
Nvdot & 0 \\
Nrdot & -1.0 \\
Nv & 0.03130 \\
Nabsvv & 3.95645 \\
Nabsrv & 0.130 \\
Nr & -1.900 \\
Nabsvr & 0.080 \\
Nabsrr & -0.750 \\
lxR1 & -0.549 \\
lxR2 & -0.549 \\
\hline
\end{tabular}

superiority of the MBD method. Then, a time-varying disturbance incorporating white noise and constant offset force is added in path-following simulations to demonstrate the effectiveness and robustness of the MBD method. The parameters of the vessel are given in [33] and listed in Table 1.

In the simulation, the parameter $\Delta$ in guidance law is chosen as $\Delta=8 \mathrm{~m}$. In the heading MPC Controller, the prediction horizon $N_{p}$ and control horizon $N_{u}$ are chosen as 70 and 30, respectively. The matrix $Q_{h}$ is an identity matrix with dimension $N_{p} \times N_{p}$, the matrix $R_{h}$ is also an identity matrix with dimension $N_{u} \times N_{u}$. In the surge speed controller, the prediction horizon $N_{p u}$ and control horizon $N_{u u}$ are chosen as 30 and 5 , respectively. The matrix $Q_{u}$ is an identity matrix with dimension $N_{p u} \times N_{p u}$; the matrix $R_{u}$ is also an identity matrix with dimension $N_{u u} \times N_{u u}$. In the surge speed controller, the vessel initial forward speed of the vessel is set at $0.5 \mathrm{~m} / \mathrm{s}$ and the other state of vessel are all set to zero. The constraints of surge force are $|\tau x| \leq 5$. The constraints of steering moment are $|\tau z| \leq 1$, and the steering moment increment is $|\Delta \tau z| \leq 0.5$.

The path-following results of different methods with no external disturbance are shown in Figure 2, and the corresponding surge speed and surge force $\tau x$ are shown in Figure 3. From the results shown in Figure 2, we can see that the path following results of different methods are very close and the actual path of the vessel converges to the reference path eventually.

In the surge speed control, as shown in Figure 3, the commanded speed is $0.8 \mathrm{~m} / \mathrm{s}$ at the first $200 \mathrm{~s}$ then changed to $0.6 \mathrm{~m} / \mathrm{s}$. All the surge control method can guarantee the surge speed converge to the command speed. Due to the way-point changed, the sway and yawing motion result in added resistance. There is some speed loss in path following 


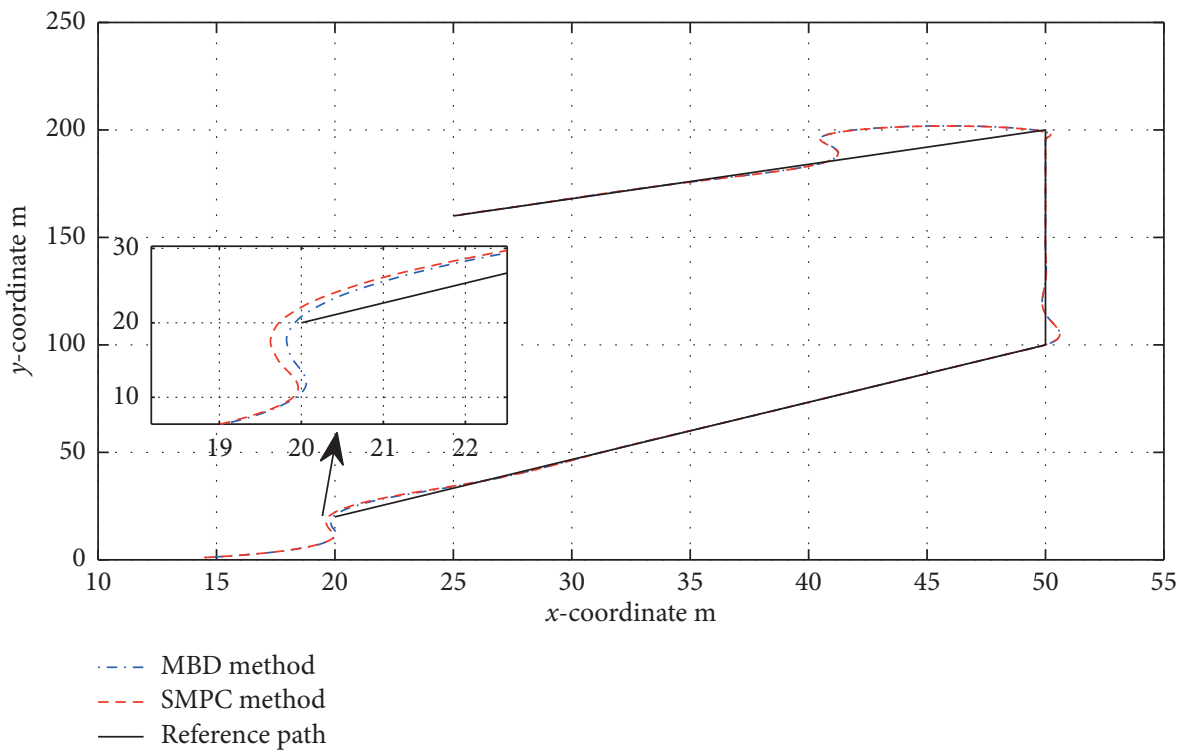

Figure 2: Path following results of different methods.

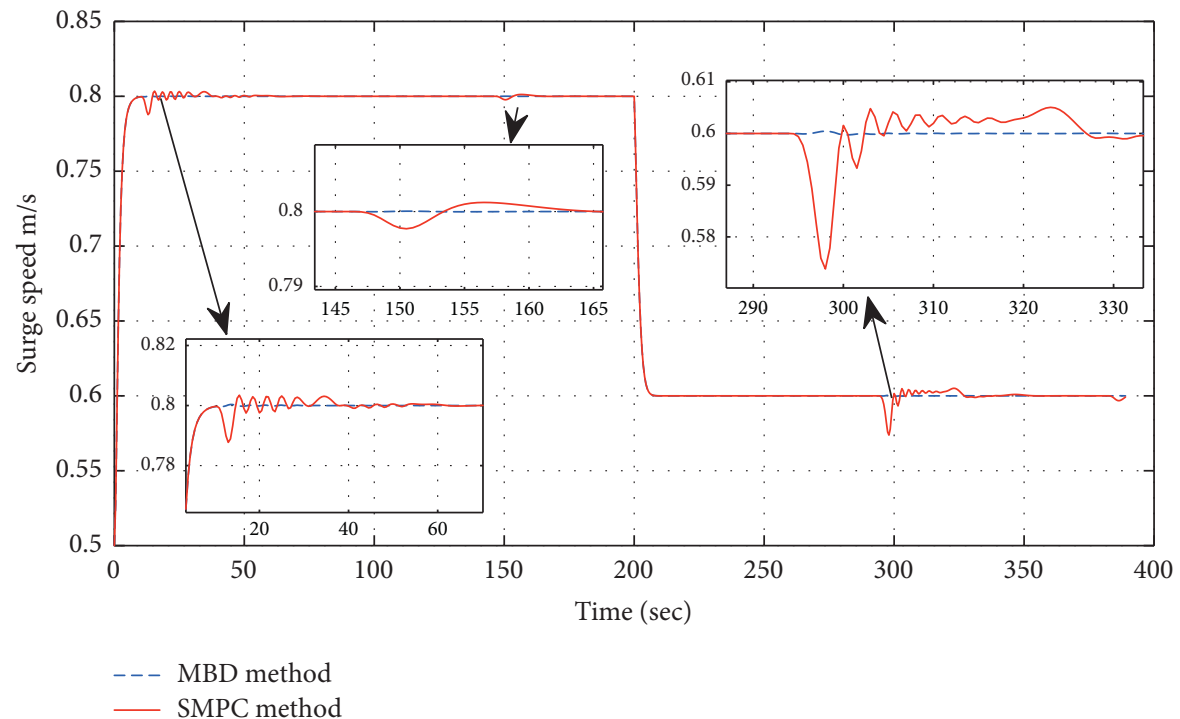

(a)

Figure 3: Continued. 


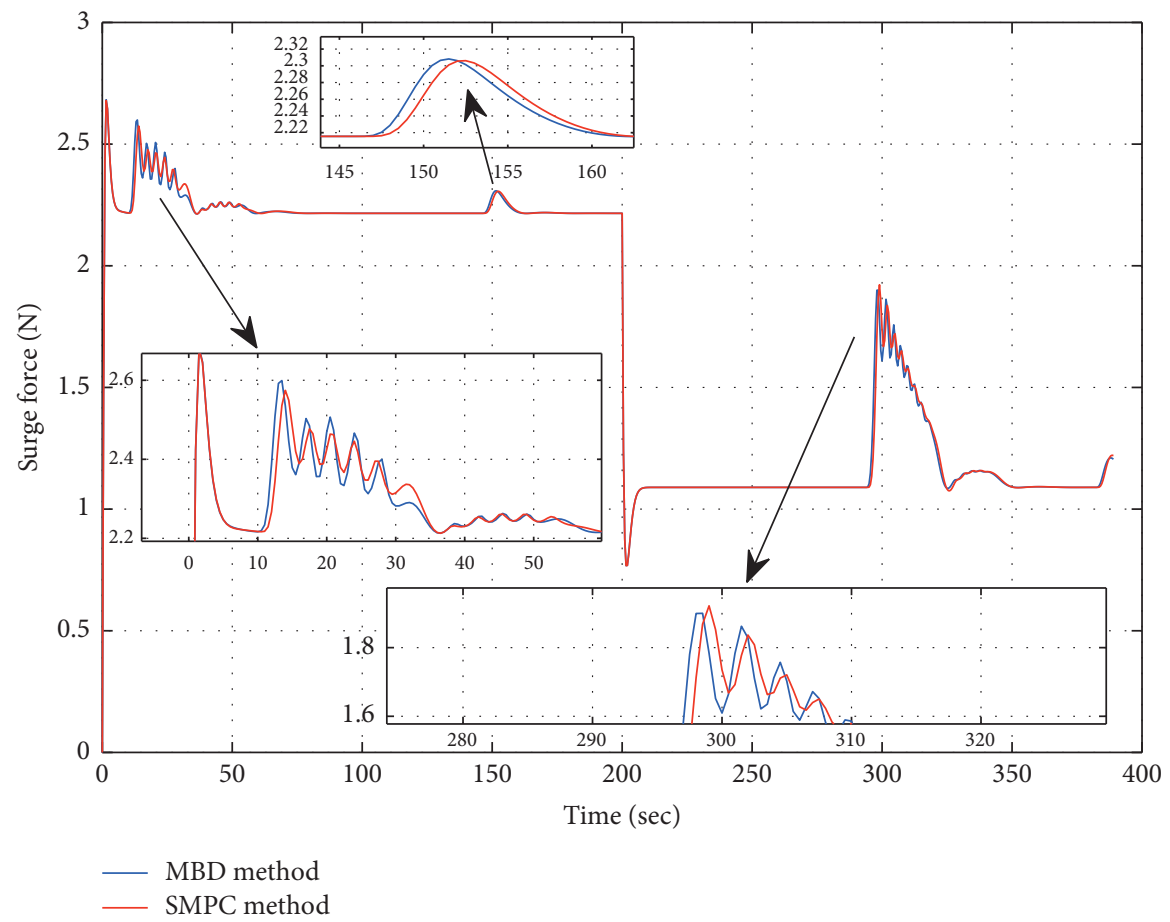

(b)

FiguRE 3: Surge speed control performance of different methods. (a) Surge speed in path following of different methods. (b) Surge force in path following of different methods.

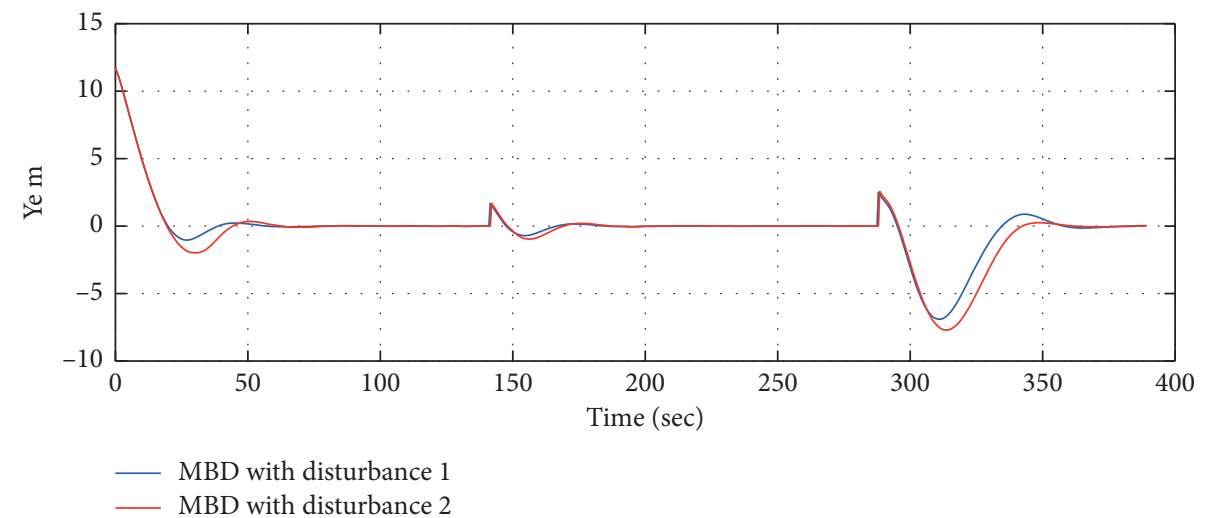

FIGURE 4: Cross-error in path following under different disturbances.

under the SMPC control method, but there are no significant fluctuations in surge speed under the control of the MBD method, see Figure 3(a)) at about 20 s, 150 s, and 300 s. From the surge force output by different methods shown in Figure 3(b)), we can see, comparing the surge force output by the MBD method and the SMPC method, that the surge force output by SMPC controller has a time delay when there is an add resistance. Moreover, the time delay of surge force is the reason why there is a significant surge speed loss in speed control.

In the MBD method, the sway and yawing motion generated by the steering moment is predicted as a time- varying disturbance, which is compensated at the speed control. Nevertheless, in SMPC control, there will be no external force to compensate disturbance until there is an offset in surge speed. So, the MBD method has a better performance than the SMPC method in heading and speed control of the vessel in path following.

To further demonstrate the effectiveness of the MBD method in the real sea environment, the path following results with time-varying disturbance is shown in Figures 4-6. The disturbance one shown in Figures 5 and 6 consists of white noise with noise power of 0.0025 , and a constant force of $0.3 \mathrm{~N}$; the disturbance two shown in 


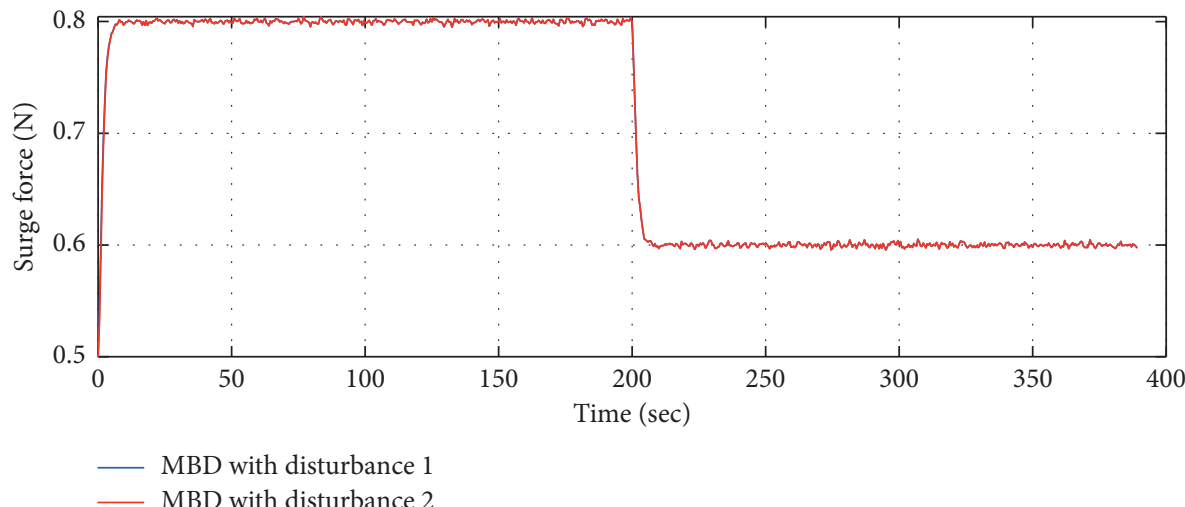

(a)

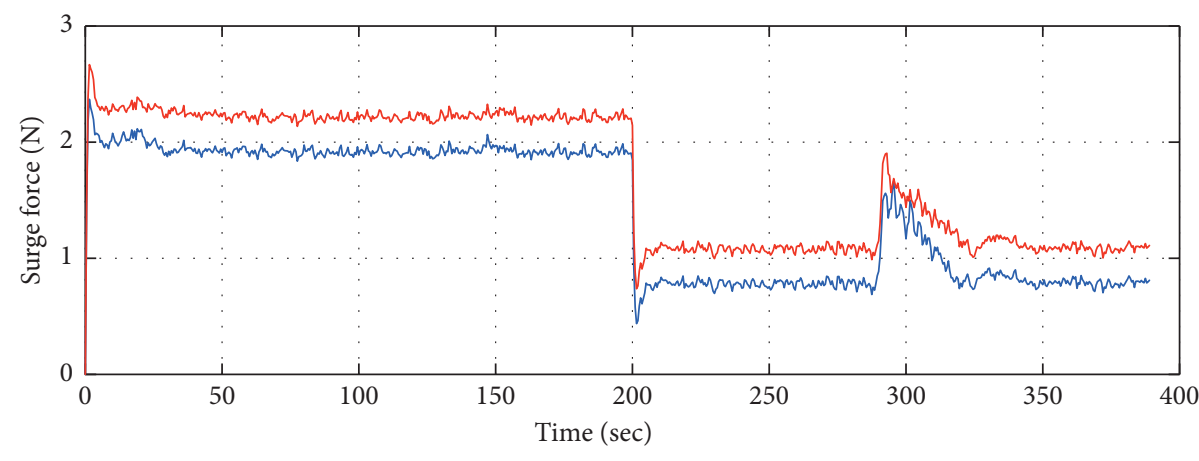

(b)

Figure 5: Surge speed in path following under different disturbances. (a) Surge speed control results under different disturbances. (b) Surge force results under different disturbances.

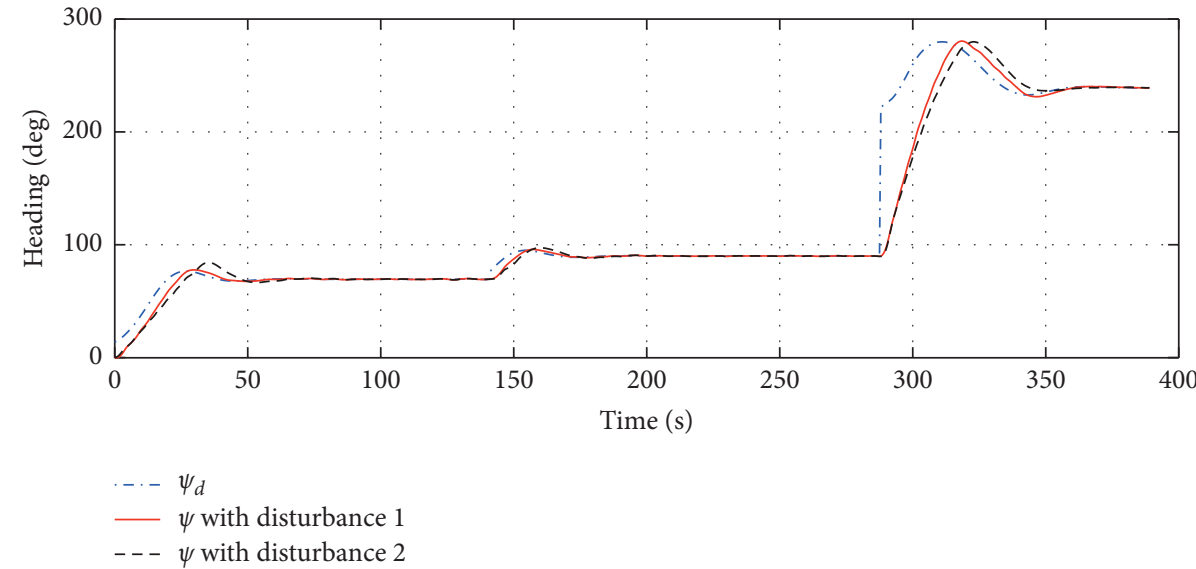

(a)

Figure 6: Continued. 


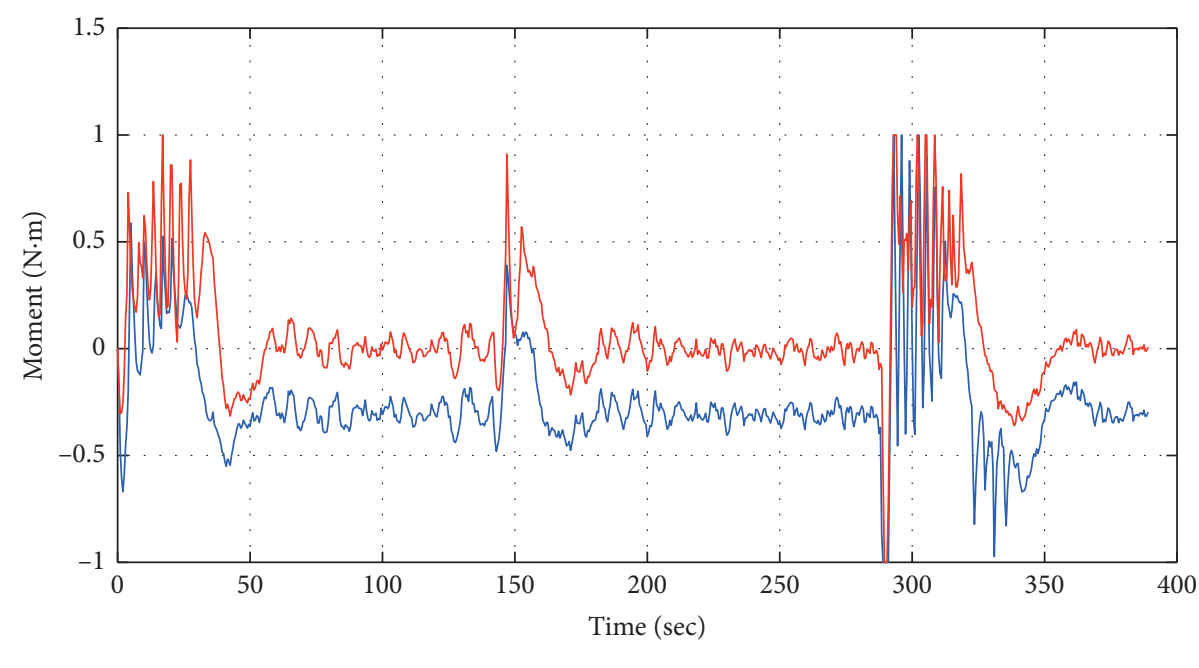

MBD with disturbance 1

- MBD with disturbance 2

(b)

FIGURE 6: Heading control results in path following under different disturbances. (a) Heading control results under different disturbances. (b) Steering moment under different disturbances.

Figures 5 and 6 is a white noise with noise power of 0.0025 .

From Figure 4, we can see that the cross error $Y_{e}$ in path following converges to zero; the addition unknown timevarying disturbance has no influence in the overall performance. The heading and speed control results shown in Figures 5 and 6 demonstrate the effectiveness of the MBD method under unknown time-varying disturbance. Also, the steering moment $\tau z$, the increment of steering moment $\Delta \tau z$, and the surge force $\tau x$ are all restricted to the constraints.

\section{Conclusions}

In this paper, to solve the surge speed loss in path following due to the added resistance generated by sway-yaw motion, the MBD method for surge speed and heading control in vessel path following is presented. In the MBD method, the heading controller and the surge speed controller can be designed under the framework of MPC, independently. The nonlinear control problem of control vessel heading and controller together can be decoupled into two MPC control problems based on the linear heading model and linear surge model, which makes the progress of designing the controller easier. The output of the heading controller is used to predict the time-varying add resistance, which is treated as timevarying disturbance and compensated in surge speed control.

Compared to the control results of the SMPC method, the surge speed has no significant fluctuations in path following under the control of the MBD method, which demonstrates the superiority of the MBD method. The pathfollowing results under the unknown time-varying disturbance demonstrate the effectiveness and robustness of the MBD method.

\section{Data Availability}

The data used to support the findings of this study are included within the article.

\section{Conflicts of Interest}

The authors of this paper declare that there are no conflicts of interest regarding the publication of this article.

\section{Acknowledgments}

This work was supported in part by the Guangdong Innovative and Entrepreneurial Research Team Program under Grant 2014ZT05G304 and National Natural Science Foundation of China under Grants 61573159 and 61273174.

\section{References}

[1] X. Wang, H. Su, X. Wang, and G. Chen, "Fully distributed event-triggered semiglobal consensus of multi-agent systems with input saturation," IEEE Transactions on Industrial Electronics, vol. 64, no. 6, pp. 5055-5064, 2016.

[2] X. L. Wang, H. Su, M. Z. Chen, X. F. Wang, and G. Chen, "Reaching non-negative edge consensus of networked dynamical systems," IEEE Transactions on Cybernetics, vol. 48, no. 9, pp. 2712-2722, 2018.

[3] X. Wang, H. Su, M. Z. Chen, and X. Wang, "Observer-based robust coordinated control of multiagent systems with input saturation," IEEE Transactions on Neural Networks and Learning Systems, vol. 29, no. 5, pp. 1933-1946, 2017.

[4] Y. Liu and H. Su, "Containment control of second-order multi-agent systems via intermittent sampled position data communication," Applied Mathematics and Computation, vol. 362, Article ID 124522, 2019. 
[5] X. Wang and H. Su, "Self-triggered leader-following consensus of multi-agent systems with input time delay," $\mathrm{Neu}$ rocomputing, vol. 330, pp. 70-77, 2019.

[6] Y. Liu and H. Su, "Some necessary and sufficient conditions for containment of second-order multi-agent systems with sampled position data," Neurocomputing, vol. 378, pp. 228237, 2020.

[7] L. Moreira, T. I. Fossen, and C. G. Soares, "Path following control system for a tanker ship model," Ocean Engineering, vol. 34, no. 14-15, pp. 2074-2085, 2007.

[8] A. M. Lekkas and T. I. Fossen, "A time-varying lookahead distance guidance law for path following," IFAC Proceedings Volumes, vol. 45, no. 27, pp. 398-403, 2012.

[9] A. M. Lekkas and T. I. Fossen, "Integral los path following for curved paths based on a monotone cubic hermite spline parametrization," IEEE Transactions on Control Systems Technology, vol. 22, no. 6, pp. 2287-2301, 2014.

[10] T. I. Fossen, K. Y. Pettersen, and R. Galeazzi, "Line-of-sight path following for dubins paths with adaptive sideslip compensation of drift forces," IEEE Transactions on Control Systems Technology, vol. 23, no. 2, pp. 820-827, 2015.

[11] L. Liu, D. Wang, and Z. Peng, "ESO-based line-of-sight guidance law for straight line path following with exact sideslip compensation," in Proceedings of the 12th World Congress on Intelligent Control and Automation (WCICA), pp. 677-681, IEEE, Guilin, China, June 2016.

[12] L. Liu, D. Wang, Z. Peng, and H. Wang, "Predictor-based los guidance law for path following of underactuated marine surface vehicles with sideslip compensation," Ocean Engineering, vol. 124, pp. 340-348, 2016.

[13] Z. Zheng and L. Sun, "Path following control for marine surface vessel with uncertainties and input saturation," Neurocomputing, vol. 177, pp. 158-167, 2016.

[14] Y. Qu, L. Cai, and H. Xu, "Curved path following for unmanned surface vehicles with heading amendment," IEEE Transactions on Systems, Man, and Cybernetics: Systems, 2019.

[15] K. D. Do, J. Pan, and Z.-P. Jiang, "Robust and adaptive path following for underactuated autonomous underwater vehicles," Ocean Engineering, vol. 31, no. 16, pp. 1967-1997, 2004.

[16] K. D. Do and J. Pan, "Robust path-following of underactuated ships: theory and experiments on a model ship," Ocean Engineering, vol. 33, no. 10, pp. 1354-1372, 2006.

[17] Z. Li, J. Sun, and S. Oh, "Design, analysis and experimental validation of a robust nonlinear path following controller for marine surface vessels," Automatica, vol. 45, no. 7, pp. 1649-1658, 2009.

[18] Z. Hui and S. Jihong, "Path following control of underactuated ship based on nonlinear backstepping," in Proceedings of the IEEE International Conference on Information and Automation (ICIA), pp. 1-6, IEEE, Yinchuan, China, August 2013.

[19] Z. Zheng and M. Feroskhan, "Path following of a surface vessel with prescribed performance in the presence of input saturation and external disturbances," IEEE/ASME Transactions on Mechatronics, vol. 22, no. 6, pp. 2564-2575, 2017.

[20] L. Yang and J. Zhang, "Path following of underactuated surface vessels based on neural sliding mode," in Proceedings of the IEEE International Conference on Mechatronics and Automation (ICMA), pp. 2261-2266, IEEE, Beijing, China, August 2015.

[21] W. Caharija, K. Y. Pettersen, M. Bibuli et al., "Integral line-ofsight guidance and control of underactuated marine vehicles: theory, simulations, and experiments," IEEE Transactions on Control Systems Technology, vol. 24, no. 5, pp. 1623-1642, 2016.
[22] M. S. Wiig, K. Y. Pettersen, and T. R. Krogstad, "Uniform semiglobal exponential stability of integral line-of-sight guidance laws," IFAC-PapersOnLine, vol. 48, no. 16, pp. 61-68, 2015.

[23] Y. Qu, H. Xu, W. Yu, H. Feng, and X. Han, "Inverse optimal control for speed-varying path following of marine vessels with actuator dynamics," Journal of Marine Science and Application, vol. 16, no. 2, pp. 225-236, 2017.

[24] Z. Peng, J. Wang, and Q.-L. Han, "Path-following control of autonomous underwater vehicles subject to velocity and input constraints via neurodynamic optimization," IEEE Transactions on Industrial Electronics, vol. 66, no. 11, pp. 8724-8732, 2018.

[25] Z. Zheng, L. Sun, and L. Xie, "Error-constrained LOS path following of a surface vessel with actuator saturation and faults," IEEE Transactions on Systems, Man, and Cybernetics: Systems, vol. 48, no. 10, pp. 1794-1805, 2017.

[26] S.-R. Oh and J. Sun, "Path following of underactuated marine surface vessels using line-of-sight based model predictive control," Ocean Engineering, vol. 37, no. 2-3, pp. 289-295, 2010.

[27] H. Zheng, R. R. Negenborn, and G. Lodewijks, “Trajectory tracking of autonomous vessels using model predictive control," IFAC Proceedings Volumes, vol. 47, no. 3, pp. 8812-8818, 2014.

[28] J. Zhang, T. Sun, and Z. Liu, "Robust model predictive control for path-following of underactuated surface vessels with roll constraints," Ocean Engineering, vol. 143, pp. 125-132, 2017.

[29] C. Shen, Y. Shi, and B. Buckham, "Integrated path planning and tracking control of an AUV: a unified receding horizon optimization approach," IEEE/ASME Transactions on Mechatronics, vol. 22, no. 3, pp. 1163-1173, 2016.

[30] B.-O. H. Eriksen and M. Breivik, "Modeling, identification and control of high-speed asvs: theory and experiments," in Sensing and Control for Autonomous Vehicles, pp. 407-431, Springer, Berlin, Germany, 2017.

[31] M. E. N. Sorensen, M. Breivik, and B.-O. H. Eriksen, "A ship heading and speed control concept inherently satisfying actuator constraints,", in Proceedings of the IEEE Conference on Control Technology and Applications (CCTA), pp. 323-330, IEEE, Mauna Lani, HI, USA, August 2017.

[32] Y. Liao, Y. Pang, and L. Wan, "Combined speed and yaw control of underactuated unmanned surface vehicles," in Proceedings of the 2nd International Asia Conference on Informatics in Control, Automation and Robotics (CAR 2010), vol. 1, pp. 157-161, IEEE, Wuhan, China, March 2010.

[33] R. Skjetne, The maneuvering problem, vol. 1, NTNU, Trondheim, Norway, 2005, Ph.D-Thesis.

[34] M. Abdelaal, M. Franzle, and A. Hahn, "Nonlinear model predictive control for tracking of underactuated vessels under input constraints," in Proceedings of the IEEE European Modelling Symposium (EMS), pp. 313-318, IEEE, Madrid, Spain, October 2015.

[35] T. I. Fossen, Handbook of Marine Craft Hydrodynamics and Motion Control, John Wiley \& Sons, Hoboken, NY, USA, 2011.

[36] D. Limon, T. Alamo, and E. Camacho, "Stable constrained mpc without terminal constraint," in Proceedings of the 2003 American Control Conference, vol. 6, pp. 4893-4898, IEEE, Denver, CO, USA, June 2003.

[37] Z. Liu, H. Jin, M. J. Grimble, and R. Katebi, "Ship forward speed loss minimization using nonlinear course keeping and roll motion controllers," Ocean Engineering, vol. 113, pp. 201-207, 2016. 